\title{
Ultrasound-guided insertion of peripherally inserted central catheter after anesthetic induction in children undergoing surgery for moyamoya disease - Thirty cases report -
}

Received December 28, 2020

Revised March 10, 2021

Accepted March 14, 2021

\section{Corresponding author}

Hee-Soo Kim, M.D., Ph.D.

Department of Anesthesiology and

Pain Medicine, Seoul National

University Hospital, 101 Daehak-ro,

Jongno-gu, Seoul 03080, Korea

Tel: 82-2-2072-3659

Fax: 82-2-745-5587

E-mail: dami0605@snu.ac.kr

This study was presented in the Korean Society of Pediatric Anesthesia Annual Meeting, 17 October 2020, online.

\section{Sang-Hwan Ji', Sol Ji Yoo' ${ }^{1}$, Sung-Ae Cho ${ }^{2}$, Young-Eun Jang ${ }^{1}$, Eun-Hee Kim ${ }^{1}$, Ji-Hyun Lee ${ }^{1}$, Jin-Tae Kim ${ }^{1,3}$, and Hee-Soo Kim ${ }^{1,3}$}

'Department of Anesthesiology and Pain Medicine, Seoul National University Hospital, Seoul, ${ }^{2}$ Department of Anesthesiology and Pain Medicine, Konyang University Hospital, Daejeon, ${ }^{3}$ Department of Anesthesiology and Pain Medicine, Seoul National University College of Medicine, Seoul, Korea

\begin{abstract}
Background: Pediatric patients with moyamoya disease are vulnerable to ischemic attacks following physical or emotional stress, such as those experienced during blood sampling. A central venous catheter might be beneficial for blood sampling, and a peripherally inserted central catheter (PICC) is a considerable option for central venous access. However, PICC insertion during anesthetic management is relatively rare.
\end{abstract}

Case: Thirty cases of ultrasound-guided PICC insertion were performed in children undergoing surgery for moyamoya disease after anesthetic induction. Positioning was successful in 22 cases, and 5 were malpositioned. In three cases, the peripheral insertion failed. Adjustment of the insertion depth was performed in nine cases. No complications related to catheterization were observed during the procedure or the catheter indwelling period.

Conclusions: We report the successful use of PICC in children undergoing surgery for moyamoya disease with a considerable success rate and low incidence of malpositioning or complications.

Keywords: Children; General anesthesia; Central venous catheterization; Moyamoya disease; Neurosurgery; Ultrasonography.
Central venous catheterization is frequently performed during general anesthesia, mainly for fluid administration and monitoring of central venous pressure. In addition, the need for frequent blood sampling can also be a reason for central venous catheterization to reduce the pain or fear of needle insertion. Since pediatric patients with moyamoya disease (MMD) are at risk of ischemic attack when crying or undergoing physical or emotional stress, a central venous catheter would be helpful for postoperative blood sampling or fluid management to prevent these events.

A peripherally inserted central catheter (PICC) is a good option for central venous access [1] with good compliance, low complication rate [2,3], and readiness for ambulation after surgery, compared to the internal jugular or subclavian veins. However, it is rarely selected by anesthesiologists in the operating room. While PICC can be inserted at the bed-

This is an Open Access article distributed under the terms of the Creative Commons Attribution Non-Commercial License (http://creativecommons.org/licenses/by-nc/4.0) which permits unrestricted non-commercial use, distribution, and reproduction in any medium, provided the original work is properly cited.

Copyright (c) the Korean Society of Anesthesiologists, 2021 
side or under fluoroscopic guidance prior to surgery for adults, PICC insertion for pediatric patients is not easy, and they require sedation for the procedure in most cases. Under general anesthesia, ultrasound is readily applicable to pediatric patients without causing radiation hazards compared to fluoroscopy, which is frequently used as a guide for PICC insertion.

We recently introduced a policy to insert the PICC under ultrasound guidance after induction of general anesthesia in pediatric patients undergoing surgery for MMD. We report our cases along with a summary of the success and complication rates.

\section{CASE REPORT}

\section{Ethics statement}

This report was approved by the Institutional Review Board of Seoul National University Hospital (no. 2004-2311119), and the requirement for obtaining informed consent was waived. The reason for the exemption from consent was as follows: First, obtaining informed consent from the patients was not feasible because they had already been discharged from the hospital at the moment we started to review the cases. Second, there was no reason to assume disagreement regarding the use of data from the patients, and there was no chance of affecting any of the patients' treatment or prognosis.

\section{Study population}

We reviewed 30 cases of ultrasound-guided PICC insertion after anesthetic induction in pediatric patients undergoing surgery for MMD between January 2020 and April 2020. The baseline characteristics are shown in Table 1. Seventeen $(56.7 \%)$ patients had previously undergone neurosurgery with central venous catheterization using one of the internal jugular veins. Patients with connective tissue disease or vascular disease other than MMD, any skin lesion in the upper extremity, and unstable vital signs on anesthetic induction were excluded from the PICC insertion.

\section{Catheter insertion technique}

After conventional induction of general anesthesia, the patient was positioned for PICC insertion in the supine position, and the right upper arm was abducted [4]. After posi- tioning, the basilic or cephalic vein was evaluated using an ultrasound device (E-CUBE i7, Alpinion Medical Systems Co., Ltd., Korea or Sonosite X-Porte, Fujifilm Sonosite, Inc., USA) to select the venipuncture site. If the basilic vein was selected, the elbow was flexed at $90^{\circ}$, while it was extended to the cephalic vein. The patient's right arm was covered with a surgical drape after sterilization. Turbo-Ject ${ }^{\circledR}$ power-injectable PICC (Cook Medical LLC, USA) with a size of 3 or 4 Fr was prepared. Even though 4-Fr catheters are recommended for veins larger than $4 \mathrm{~mm}$ in diameter [5], a larger catheter size may be related to a higher risk of thrombosis [6]. In most cases, we used 3-Fr catheters in an effort to use a catheter as small as possible to prevent thrombosis. Catheters sized 4 Fr were used only for patients weighing $>70 \mathrm{~kg}$. Before venipuncture, the length of catheter insertion was determined by measuring the distance between the targeted venipuncture site and the sternal notch [7]. The catheter was then trimmed according to the required length. The targeted vein was punctured under ultrasound guidance, followed by the insertion of a 20-gauge intravenous $\mathrm{BD}$ angiocath ${ }^{\mathrm{TM}}$ (Becton, Dickinson, and company Korea, Korea) catheter. To ensure stability, we tried to maintain a distance of at least 4 $\mathrm{cm}$ between the antecubital fossa and venipuncture site [8]. Subsequently, a guidewire was inserted via an intravenous catheter. An introducer was inserted along the guidewire after a minimal skin incision was made at the venipuncture site. After removal of the guidewire, the PICC was inserted through the introducer and advanced until the entire catheter was inserted. During the advancement of the catheter,

Table 1. Baseline Characteristics of Subjects, Selected Veins, and Catheter Size for Insertion of a Peripherally-inserted Central Catheter

\begin{tabular}{lc}
\hline \multicolumn{1}{c}{ Characteristic } & Value \\
\hline Sex $(\mathrm{M} / \mathrm{F})$ & $20 / 10$ \\
Age $(\mathrm{yr})$ & $7.5(6,9)$ \\
Height $(\mathrm{cm})$ & $124.0(115.7,146.2)$ \\
Weight $(\mathrm{kg})$ & $28.4(21.7,46.6)$ \\
Selected vein & \\
$\quad$ Basilic & $21(70.0)$ \\
Cephalic & $6(20.0)$ \\
$\quad$ Failure for both & $3(10.0)$ \\
Vein diameter (mm) & $4.0 \pm 0.9$ \\
Vein depth (mm) & $5.4(4.1,8.5)$ \\
Catheter size (Fr) & \\
3 & $26(86.7)$ \\
4 & $4(13.3)$ \\
\hline
\end{tabular}

Values are represented as number (\%), median (1Q, 3Q), or mean \pm SD. 
the patient's head was turned to the ipsilateral side. The arm was then moved cephalad in an effort to facilitate successful insertion [9]. The function of the PICC was tested by aspiration of blood and flushing with normal saline through the catheter.

\section{Confirmation of the catheter tip}

Immediately after insertion of the catheter, the right internal jugular vein was traced using ultrasound to confirm that the catheter did not enter the right internal jugular vein. When the catheter was observed in the right internal jugular vein, the catheter was withdrawn slightly and then reinserted until the catheter was completely inserted but not visible in the right internal jugular vein. If possible, the right subclavian vein was also visualized to ensure that the catheter had not migrated into the right innominate vein.

After the end of the surgery, the position of the tip of the catheter was checked via chest radiography (CXR) at the intensive care unit or the post-anesthesia care unit. The tip position was classified into three categories: "Optimal" for those located within a $3-\mathrm{cm}$ margin from the cavo-atrial junction, "suboptimal" if they were within the superior vena cava (SVC) or the right atrium, but located outside the $3-\mathrm{cm}$ margin from the cavo-atrial junction, or "malpositioned" in cases involving vessels at locations other than the SVC [10]. We defined success as the optimal or suboptimal position of the catheter tip in the postoperative CXR.

\section{Statistical analysis}

Demographic data, history of previous surgery, vein selected for puncture, depth from skin and diameter of the selected vein, determined insertion length of the catheter, the success rate at the first attempt, overall success rate, number of insertion attempts, reasons for failed attempts, postoperative repositioning of the catheter, duration of postoperative catheter indwelling, and functioning of the catheter were reviewed. Immediate and long-term complications, including hematoma formation, thrombosis, infection, insertion site oozing, skin reactions, and catheter migration, were also reviewed. The Pearson correlation coefficient between the patient height and the determined insertion length of the catheter was calculated. Statistical analyses were performed using SPSS $^{\circledR}$ statistics version 23.0 (IBM, USA).

\section{Outcomes of PICC insertion}

Among the 30 patients, the procedure was completed in $27(90.0 \%)$ patients, while we failed to introduce the catheter in the other 3 patients. Twenty-two (73.3\%) cases showed successful PICC with optimal or suboptimal positioning of the catheter tip on confirmation with CXR. Insertion was successful in the first attempt in 19 patients. Detailed information on the insertion attempts is presented in Table 2.

Upon confirmation with postoperative CXRs for the completed cases, 5 (16.7\%) cases showed malpositioning of the catheter, 3 in the right internal jugular vein, and 2 in the left brachiocephalic vein. Fig. 1 shows examples of CXRs for the successful placement and malpositioning of the catheter tip. Among the malpositioned cases, the basilic vein was selected in four cases and the cephalic vein was selected in one case. In one case of malpositioning of the internal jugular vein, the PICC was revised in the angiography room the day after. In another case of malpositioning of the internal jugular vein, the catheter was withdrawn by $4 \mathrm{~cm}$. In the remaining 3 malpositioned cases, the catheter was used without repositioning. In 6 cases, the catheter tip was located deeper than expected. In these cases, the catheter was withdrawn by a median $(1 \mathrm{Q}, 3 \mathrm{Q})$ of $3.8(3.1,5.5) \mathrm{cm}$. Two of the withdrawn cases involved follow-up CXRs, which confirmed the withdrawal of the catheter to the proximal end of the right

Table 2. Results of Peripherally Inserted Central Catheter Insertion

\begin{tabular}{lc}
\multicolumn{1}{c}{ Subject } & Value \\
\hline Catheter insertion length $(\mathrm{cm})$ & $28(26,32)$ \\
Number of attempts to success & \\
1 & $19(63.3)$ \\
2 & $6(20.0)$ \\
3 & $2(6.7)$ \\
Reason for failed attempts $(\mathrm{n}=17)$ & \\
Venipuncture failure & $3(17.6)$ \\
Guidewire insertion failure & $2(11.8)$ \\
Dilation failure & $3(17.6)$ \\
Catheter advancement failure & $9(52.9)$ \\
Catheter tip position & \\
Optimal* $^{*}$ & $14(46.7)$ \\
Suboptimal & \\
Malpositioning & \\
Failure $^{\ddagger}$ & $8(26.7)$ \\
\hline
\end{tabular}

Values are represented as median (1Q, 3Q) or number (\%). * Located within a 3-cm margin from the cavoatrial junction. ${ }^{\dagger}$ Located within the superior vena cava (SVC) or the right atrium and could be easily repositioned by the withdrawal of the catheter. ${ }^{\ddagger}$ Locations other than the SVC. ${ }^{\S}$ Failed to insert the catheter. 

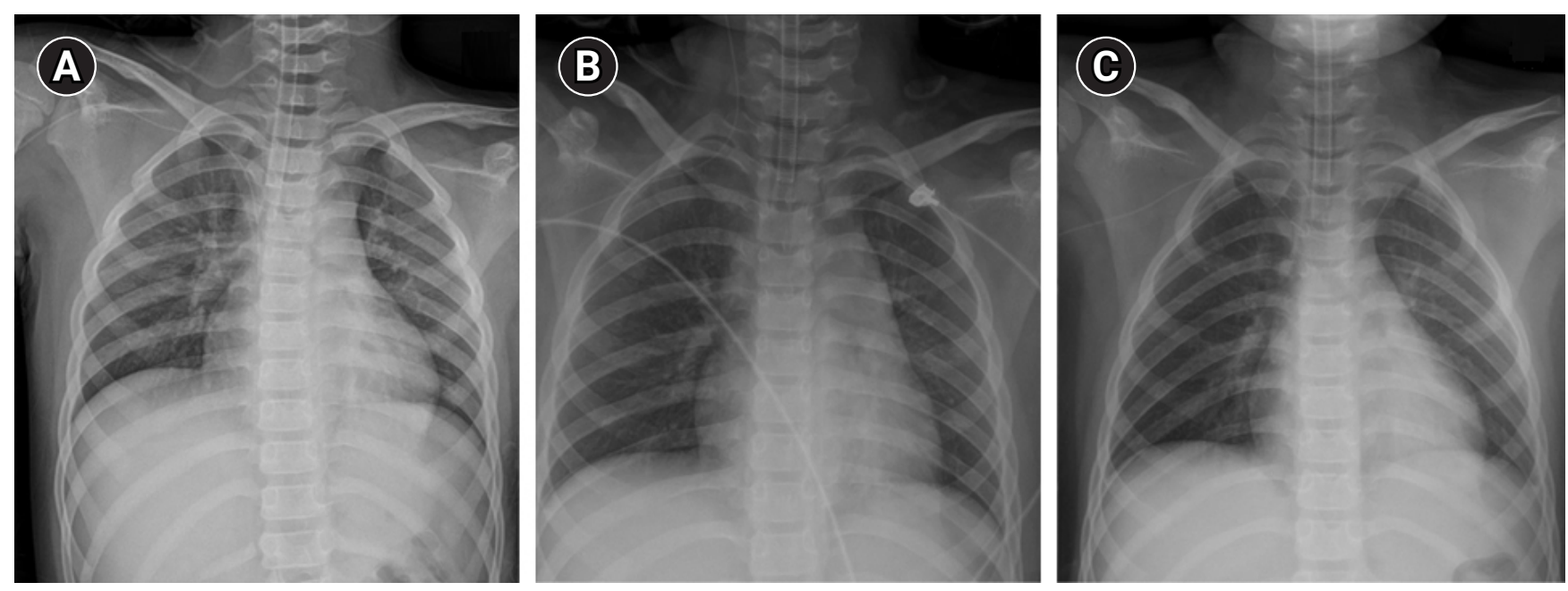

Fig. 1. Examples of postoperative chest radiographs for confirmation of the tip of peripherally inserted central catheters. The tip is located at the superior vena cava $(A)$, ipsilateral internal jugular vein (B), and contralateral brachiocephalic vein (C).

subclavian vein. In one case, the insertion length was shorter than expected to an extent of $5 \mathrm{~cm}$, and there was no adjustment.

In 3 patients, the catheter was unexpectedly withdrawn during transportation of the patient from the operating room to the intensive care unit, while re-insertion of the catheter was not performed. All successful cases showed intact catheter function, with no reported immediate complications such as hematoma, thrombosis, skin irritation, or bleeding.

The median (1Q, 3Q) duration of indwelling was $7(6,8)$ days, and the total indwelling time for all subjects was 184 PICC-days with no reported complications or complaints of discomfort. There was a strong positive correlation between the patient's height and the insertion length of the catheter, with a Pearson correlation coefficient of $0.906(\mathrm{P}<0.001)$.

Four patients were included twice in our data, and all showed successful insertion during the first operation. In the second operation, the four cases showed a failure of PICC insertion, malpositioning to the contralateral side, successful insertion in the same vein, and successful insertion in the alternate vein (cephalic-basilic).

We did not survey patient satisfaction with PICC after surgery. However, neurosurgeons in charge of their in-hospital care reported an overall increase in patients' comfort and stability during the hospital stay with PICC compared to access via the internal jugular or subclavian veins.

\section{DISCUSSION}

We summarized cases of PICC insertion under ultrasound guidance after induction of general anesthesia in pediatric patients undergoing surgery for MMD with successful positioning of the catheter tip in 22 out of 30 cases.

In recent studies, success rates defined as proper positioning of the PICC catheter with ultrasound-guided insertion have been reported to be about $81.2 \%$ and $94.4 \%[10,11]$ in adults and $83.9 \%$ [12] in children. Our success rate of $73.3 \%$ was relatively lower than these results. In our cases, catheter insertion was performed during anesthesia and prior to surgery. As we just introduced the policy of PICC insertion in our department, the proficiency of the practitioner might be a cause for the lower success rate. Moreover, since we reviewed only a small number of cases, the success rate may be inconsistent with previous data. Among the three cases of failed insertion, it was difficult to advance the catheter into the right subclavian vein with or without the obturator in two cases, and advancement of the introducer was impossible in the other case. Reports of common reasons for PICC insertion failure are difficult to find in the literature, so we could not compare our results with those of previous studies.

The incidence of malpositioning of the catheter tip is reported to be higher with ultrasound guidance than with fluoroscopic guidance [13] and varies from $8.4 \%$ to $27 \%[10,13]$. In our study, the malpositioning rate of the inserted cases was $18.5 \%$, which is consistent with these results. Although we checked via ultrasound immediately after catheter placement, upper limb PICC in children may move with arm movement to an extent of 2.2 rib spaces [14], which can be an explanation for this malpositioning. Abduction of the right upper arm during the procedure might have affected the migration of the 
catheter tip since the arm was abducted during the surgery and immediate postoperative period. We might have had a better agreement between CXR and ultrasound if CXR was checked immediately after insertion. However, since we wanted to avoid exposing patients to additional radiation, we decided not to check CXR immediately, but to rely on ultrasound and routine postoperative CXR. This was possible because our primary reason for PICC insertion was acquiring a stable route of blood sampling and fluid administration, but not monitoring the central venous pressure.

Immediate complications associated with PICC insertion are reported to be rare, with a global complication rate of $30.2 \%$ during indwelling and 11.1 per 1000 PICC-days [15]. In our study group, there were no records of any complications associated with PICC. In contrast, in our previous study, patients maintained the PICC for a median of 17 days, ranging from 2 to 174 days [15], and the meantime to the onset of complications was 16.1 days. The relatively short catheter maintenance period may have affected our complication-free outcomes. In three cases, however, accidental withdrawal of the catheter occurred during transportation of the patient, which indicated the need for more caution.

Although some reports have described landmark-based determination of PICC insertion length, they are limited to adults [7]. Since there is no standardized method to determine the appropriate length of PICC insertion in children and no fluoroscopy was available, we simply measured the distance between the targeted insertion site and the sternal notch [7]. The insertion length was optimal in approximately half of the cases and was acceptable (optimal and suboptimal combined) in $81.5 \%$ of the inserted cases. Although there is one report on the landmark-based determination of the length of PICC in children with preliminarily taken CXRs [16], we did not employ this technique because of the absence of images that cover the right arm and the chest altogether in our institute.

While PICC insertion helps patients in postoperative management in many ways, it does not change or help in anesthetic management during surgery. From the anesthesiologists' perspective, the advantage of PICC is the relatively high safety compared to other central venous catheterization methods.

Our study had some limitations. First, as we only included elective surgeries for MMD and patients were relatively healthy except for that, our cases had a relatively short indwelling duration of PICC and a low complication rate. These may be valuable data, but the findings are not gener- alizable to patients with worse general conditions or those requiring extended indwelling. Further studies including other populations are needed to obtain more generalized data. Second, as patient satisfaction was not adequately recorded and compared to that for conventional central venous catheterization, we cannot claim that PICC is more patient-friendly than conventional insertions. However, since the median duration of indwelling was approximately 7 days, it is credible that the insertion site of the forearm will be much more comfortable to maintain than the neck or chest. A prospective study comparing PICC and other methods of central venous access will provide an answer to this concern. Third, the elapsed time of insertion was not recorded, even though there were some cases with multiple attempts. Further prospective studies are required to include the time measurements.

In conclusion, PICC can be successfully introduced to pediatric patients undergoing surgery for MMD during anesthetic management without serious complications. Further prospective studies including insertion depth determination, insertion technique, and comparison with other forms of central venous access will be needed to improve our clinical practice for pediatric patients who require central venous access.

\section{CONFLICTS OF INTEREST}

No potential conflict of interest relevant to this article was reported.

\section{DATA AVAILABILITY STATEMENT}

The datasets generated during and/or analyzed during the current study are available from the corresponding author on reasonable request.

\section{AUTHOR CONTRIBUTIONS}

Conceptualization: Hee-Soo Kim. Data curation: SangHwan Ji, Sol Ji Yoo, Hee-Soo Kim. Formal analysis: Sol Ji Yoo, Eun-Hee Kim, Ji-Hyun Lee. Methodology: Young-Eun Jang, Jin-Tae Kim. Investigation: Sang-Hwan Ji, Sung-Ae Cho, EunHee Kim, Hee-Soo Kim. Software: Sung-Ae Cho, Young-Eun Jang, Eun-Hee Kim. Writing - original draft: Sang-Hwan Ji, Sol Ji Yoo, Sung-Ae Cho, Hee-Soo Kim. Writing - review \& editing: Sang-Hwan Ji, Young-Eun Jang, Eun-Hee Kim, JiHyun Lee, Jin-Tae Kim, Hee-Soo Kim. 


\section{ORCID}

Sang-Hwan Ji, https://orcid.org/0000-0001-6736-4464

Sol Ji Yoo, https://orcid.org/0000-0002-3860-1181

Sung-Ae Cho, https://orcid.org/0000-0002-1519-3787

Young-Eun Jang, https://orcid.org/0000-0002-7511-4104

Eun-Hee Kim, https://orcid.org/0000-0003-0697-1935

Ji-Hyun Lee, https://orcid.org/0000-0002-8384-8191

Jin-Tae Kim, https://orcid.org/0000-0002-3738-0081

Hee-Soo Kim, https://orcid.org/0000-0002-2661-7944

\section{REFERENCES}

1. Schwengel DA, McGready J, Berenholtz SM, Kozlowski LJ, Nichols DG, Yaster M. Peripherally inserted central catheters: a randomized, controlled, prospective trial in pediatric surgical patients. Anesth Analg 2004; 99: 1038-43.

2. Westergaard B, Classen V, Walther-Larsen S. Peripherally inserted central catheters in infants and children - indications, techniques, complications and clinical recommendations. Acta Anaesthesiol Scand 2013; 57: 278-87.

3. Abedin S, Kapoor G. Peripherally inserted central venous catheters are a good option for prolonged venous access in children with cancer. Pediatr Blood Cancer 2008; 51: 251-5.

4. Chrisman HB, Omary RA, Nemcek AA, Ryu RK, Saker MB, Vogelzang RL. Peripherally inserted central catheters: guidance with use of US versus venography in 2,650 patients. J Vasc Interv Radiol 1999; 10: 473-5.

5. Pittiruti M, Brutti A, Celentano D, Pomponi M, Biasucci DG, Annetta MG, et al. Clinical experience with power-injectable PICCs in intensive care patients. Crit Care 2012; 16: R21.

6. Wall C, Moore J, Thachil J. Catheter-related thrombosis: a practical approach. J Intensive Care Soc 2016; 17: 160-7.

7. Venkatesan T, Sen N, Korula PJ, Surendrababu NR, Raj JP, John $\mathrm{P}$, et al. Blind placements of peripherally inserted antecubital central catheters: initial catheter tip position in relation to carina. Br J Anaesth 2007; 98: 83-8.
8. van Boxtel AJH. Maneuvers, precautions, and tricks for PICC positioning procedure. In: Peripherally inserted central venous catheters. Edited by Sandrucci S, Mussa B: Milano, Springer. 2014, p 55.

9. Ragasa J, Shah N, Watson RC. Where antecubital catheters go: a study under fluoroscopic control. Anesthesiology 1989; 71: $378-80$.

10. Kwon S, Son SM, Lee SH, Kim JH, Kim H, Kim JY, et al. Outcomes of bedside peripherally inserted central catheter placement: a retrospective study at a single institution. Acute Crit Care 2020; 35: 31-7.

11. Kim YO, Chung CR, Gil E, Park CM, Suh GY, Ryu JA. Safety and feasibility of ultrasound-guided placement of peripherally inserted central catheter performed by neurointensivist in neurosurgery intensive care unit. PLoS One 2019; 14: e0217641.

12. Jumani K, Advani S, Reich NG, Gosey L, Milstone AM. Risk factors for peripherally inserted central venous catheter complications in children. JAMA Pediatr 2013; 167: 429-35.

13. Glauser F, Breault S, Rigamonti F, Sotiriadis C, Jouannic AM, Qanadli SD. Tip malposition of peripherally inserted central catheters: a prospective randomized controlled trial to compare bedside insertion to fluoroscopically guided placement. Eur Radiol 2017; 27: 2843-9.

14. Connolly B, Amaral J, Walsh S, Temple M, Chait P, Stephens D. Influence of arm movement on central tip location of peripherally inserted central catheters (PICCs). Pediatr Radiol 2006; 36: $845-50$

15. Grau D, Clarivet B, Lotthé A, Bommart S, Parer S. Complications with peripherally inserted central catheters (PICCs) used in hospitalized patients and outpatients: a prospective cohort study. Antimicrob Resist Infect Control 2017; 6: 18.

16. Ramamurthi A, Chick JFB, Srinivasa RN, Hage AN, Grove JJ, Gemmete JJ, et al. Chest radiograph measurement technique facilitates accurate bedside peripherally inserted central catheter placement in children. Cardiovasc Intervent Radiol 2018; 41: 443-8. 